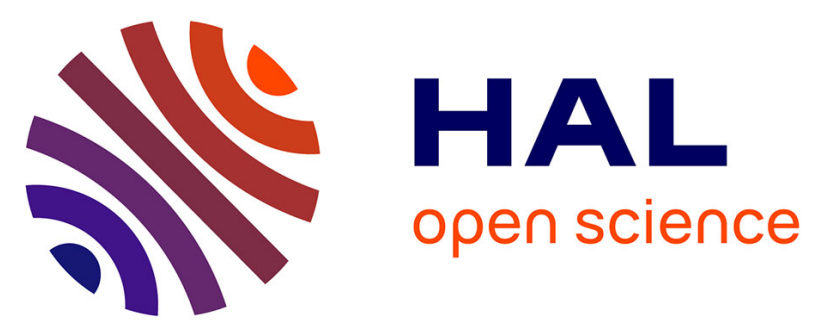

\title{
From random inhomogeneities to periodic nanostructures induced in bulk silica by ultrashort laser
}

Anton Rudenko, Jean-Philippe Colombier, Tatiana Itina

\section{To cite this version:}

Anton Rudenko, Jean-Philippe Colombier, Tatiana Itina. From random inhomogeneities to periodic nanostructures induced in bulk silica by ultrashort laser. Physical Review B: Condensed Matter and Materials Physics (1998-2015), 2016, 93 (7), pp.075427. 10.1103/PhysRevB.93.075427. ujm01340985

\section{HAL Id: ujm-01340985 \\ https://hal-ujm.archives-ouvertes.fr/ujm-01340985}

Submitted on 4 Jul 2016

HAL is a multi-disciplinary open access archive for the deposit and dissemination of scientific research documents, whether they are published or not. The documents may come from teaching and research institutions in France or abroad, or from public or private research centers.
L'archive ouverte pluridisciplinaire HAL, est destinée au dépôt et à la diffusion de documents scientifiques de niveau recherche, publiés ou non, émanant des établissements d'enseignement et de recherche français ou étrangers, des laboratoires publics ou privés. 


\title{
From random inhomogeneities to periodic nanostructures induced in bulk silica by ultrashort laser
}

\author{
Anton Rudenko, ${ }^{*}$ Jean-Philippe Colombier, ${ }^{\dagger}$ and Tatiana E. Itina ${ }^{\ddagger}$ \\ Univ Lyon, UJM-St-Etienne, Laboratoire Hubert Curien, CNRS UMR 5516, F-42000, Saint-Etienne, France
}

(Received 1 December 2015; revised manuscript received 1 February 2016; published 17 February 2016)

\begin{abstract}
Femtosecond laser-induced volume nanograting formation is numerically investigated. The developed model solves nonlinear Maxwell's equations coupled with multiple rate free carrier density equations in the presence of randomly distributed inhomogeneities in fused silica. As a result of the performed calculations, conduction band electron density is shown to form nanoplanes elongated perpendicular to the laser polarization. Two types of nanoplanes are identified. The structures of the first type have a characteristic period of the laser wavelength in glass and are attributed to the interference of the incident and the inhomogeneity-scattered light waves. Field components induced by coherent multiple scattering in directions perpendicular to the laser polarization are shown to be responsible for the formation of the second type of structures with a subwavelength periodicity. In this case, the influence of the inhomogeneity concentration on the period of nanoplanes is shown. The calculation results not only help to identify the physical origin of the self-organized nanogratings, but also explain their period and orientation.
\end{abstract}

DOI: 10.1103/PhysRevB.93.075427

\section{INTRODUCTION}

A multiple irradiation of bulk fused silica by ultrashort laser pulses is known to induce volume nanogratings (VNGs) in fused silica [1-8] and a few other materials [9-15]. First observed in 2003 by using a fixed focused femtosecond laser beam [1], these nanoplanes are found to be oriented perpendicular to the laser polarization. Nowadays, the VNGs are still considered to be the smallest embedded structures ever created by light. Further experimental investigations of the phenomenon revealed numerous interesting features of these nanogratings. In particular, it was found that laser electric field direction controls their alignment for any scan direction [2], enabling the imaging of complex polarization states [16] and the inscription of rotated nanogratings [17]. The nanoplanes are spaced by half of the wavelength in the medium independently of pulse energy $[2,8,11]$. The period was shown to decrease continuously with the number of the applied pulses $[1,8,10,13,18,19]$. It was also demonstrated that the periodicity could be controlled by changing the temporal pulse envelope [6]. A rigorous study of the phenomenon was performed by Taylor et al., where three regimes of femtosecond laser dielectric modification at different laser conditions were underlined [4]: (i) smooth modification, (ii) birefringent modification enabling nanograting self-organization, and (iii) disruptive modification at higher pulse energy and longer pulse duration. An ability to influence the resulting properties of femtosecond laser-written nanoplanes by changing laser parameters has opened up new opportunities in direct writing [3], developing polarization-sensitive devices [20-22], fabrication of nanofluidic channels [23-25], and five-dimensional optical data storage [26]. Several experimental works then investigated the evolution of nanograting formation [7,19,27,28]. A closer examination revealed that the periodically arranged nanoplanes are preferentially formed at the interface between the regions affected and unaffected by the femtosecond

\footnotetext{
*anton.rudenko@univ-st-etienne.fr

$\dagger$ jean.philippe.colombier@univ-st-etienne.fr

tatiana.itina@univ-st-etienne.fr
}

laser irradiation [7]. It was evidenced that predistributed nanogrooves and laser-induced defects strongly affected local field arrangement [27].

Several hypotheses were proposed to explain the organization of periodic structures as an interference between the incident wave and electron plasma waves $[1,13]$, an interplay between nanoplasmonic and incubation effects [2,4,29,30], self-trapping of excitons [31,32], standing wave [33], ionization scattering instabilities [34-36], second harmonic generation [37], Boson condensation [38], Coulomb explosion [39], excitation of surface plasmon polaritons $[40,41]$, and a spacecharge built from ponderomotive force [42]. The first model for nanograting formation was proposed by Shimotsuma et al. [1] based on the interference of the laser field with the laserinduced plasma waves. In this model, the nanograting period depends on the optical properties of the plasma and on the local temperature. This approach becomes invalid at a high plasma density and does not agree with the experimental observations denying the strong pulse energy dependence of the nanograting period. Another scenario was proposed by Taylor et al. [4], describing the evolution of spherically shaped nanoplasmas from the hot spots organized inhomogeneously around defects or color centers, leading to anisotropic multiphoton ionization. The periodicity is defined by the lowest order optical mode of multiple nanoplasmas, which behave like planar metallic waveguides [2]. Buividas et al. proposed that nanoplanes were pinned to the smallest possible standing wave cavity inside material [33]. Despite the predicted grating period of half the wavelength in the media agrees with experimental results, the dependency of the period on the number of laser pulses deviates from the above prediction. In fact, this dependency cannot be explained by corresponding changes of the refractive index [43]. It was also reported that longitudinal periodicity of nanogratings could be explained by interference of short-living exciton polaritons [31,32]. In contrast to plasma wave [1] and nanoplasmonic models [2,4], this approach requires low electron densities, as at higher densities the exciton-polariton interaction is heavily screened by the electron plasma when it begins to efficiently absorb laser energy [44]. Finally, Liao et al. suggested that excitation of standing plasma waves 
at the interfaces between modified and unmodified areas played a crucial role in promoting the growth of periodic nanogratings [7] and their self-organization mechanism had similarities with femtosecond-laser-induced surface ripples formation [41]. However, it was further underlined that the evidence of the defect-assisted local field rearrangement excluded the scenario that the nanograting was a result of interference between the writing beam and the surface plasma waves [27].

Furthermore, several numerical models were developed to investigate femtosecond laser-induced modification in fused silica. Some of them were based on the nonlinear Schrödinger equation (NLSE) [45-47], others used Maxwell's equations coupled with rate equation for free electron generation [34,35,41,48-51]. However, the NLSE being an asymptotic parabolic approximation of Maxwell's equations, requires the unidirectionality of the light beam and cannot describe cases where dense electron plasma is generated causing light scattering to large angles [35,36]. Most of the previous numerical models based on Maxwell's equations treated material as homogeneous media to analyze the spatial and temporal distributions of the electron density in the dielectric materials. Finally, Buschlinger et al. proposed that nanometer-sized inhomogeneities played an important role in volume nanograting formation and they were required as the seeds to start the process [48].

There were experimental evidences that the initial nonirradiated defects were present in fused silica and they were activated by femtosecond laser irradiation [7,27,31,52-57], leading to the inhomogeneous localization of the multiphoton ionization $[2,48]$. The created nanoplasma affected the laser propagation pulse by pulse due to the presence of a feedback mechanism based on memory of previous nonlinear ionization [58]. Additionally, impurities that were embedded in bulk fused silica also contributed to nanograting formation. They also enhanced and improved the nanoplasma incubation process [15,59-61]. In previous studies, the dynamics of laser interaction with inhomogeneities of different nature was studied for voids [48], for nanospheres of densified fused silica [41], and for metallic nanoparticles [62]. In all these cases, multiphoton absorption led to the generation of free electron plasma in the near field of the inhomogeneity and to the reinforcement of scattering, which induced new plasma formation in the backward propagation direction. In a recent work, it was shown that the nanometric void inhomogeneities, irradiated by a femtosecond laser, acted as the seeds of plasma structures growing against the direction of laser propagation [48]. The orientation of these nanoplanes was found to follow laser polarization direction. This result was in a good agreement with several experimental observations $[16,17]$. However, the influence of the concentration of inhomogeneities on the evolution of nanogratings, to our knowledge, has not been investigated previously, leaving unexplained the results of several experiments [15,59-61].

Despite numerous previous studies, the mechanism of VNG formation is still far from being completely understood. None of the hypotheses presented above explained the whole physics of the phenomenon and very few attempts were made to prove or to invalidate any of them based on numerical calculations [35,36,41,48,49].
Herein we present a numerical model based on Maxwell's equations coupled with a multiple rate carrier electron density equation [63], which accounts for both electronimpact (avalanche) and photoionization processes [64]. We consider smooth and sharp concentration profiles of randomly distributed inhomogeneities and identify the organized nanoplanes of two types: LFNGs (low-frequency nanogratings) and HFNGs (high-frequency nanogratings). Then we study the evolution of the nanogratings by varying several parameters, such as laser wavelength, concentration of inhomogeneities, laser pulse energy, polarization, and pulse width. Finally, we compare the obtained calculation results with the available experimental findings and discuss the physical origin of the laser-produced VNGs.

\section{NUMERICAL MODEL}

To model the laser light propagation we solve Maxwell's equations

$$
\begin{aligned}
\frac{\partial \vec{E}}{\partial t} & =\frac{\nabla \times \vec{H}}{\epsilon_{0}}-\frac{1}{\epsilon_{0}}\left(\vec{J}_{D}+\vec{J}_{\text {Kerr }}+\vec{J}_{\mathrm{pi}}\right), \\
\frac{\partial \vec{H}}{\partial t} & =-\frac{\nabla \times \vec{E}}{\mu_{0}},
\end{aligned}
$$

where $\vec{E}$ is the electric field, $\vec{H}$ is the magnetic field, $\vec{J}_{D}$ is current derived from the Drude model for the dispersive media, $\vec{J}_{\text {Kerr }}$ is Kerr polarization current, and $\vec{J}_{\text {pi }}$ is the multiphoton ionization term by using the finite-difference time-domain (FDTD) method for nonlinear and disperse media [34,35,4851]. At the edges of the grid, absorbing boundary conditions related to convolutional perfect matched layers (CPML) are set to avoid nonphysical reflections [65]. The system of nonlinear Maxwell-Ampere equations is solved by the iteration method proposed in [66].

Furthermore, the heating of the conduction band electrons is modeled by the Drude model with a time-dependent carrier density as follows:

$$
\frac{\partial \vec{J}_{D}}{\partial t}=-v_{e} \vec{J}_{D}+\frac{e^{2} n_{e}}{m_{e}} \vec{E},
$$

where $e$ is the elementary charge, $m_{e}$ is the electron mass, $n_{e}$ is the free carrier density, and $v_{e}=2 \times 10^{15} \mathrm{~s}^{-1}$ is the electron collision frequency [67-70]. In addition, a nonlinearity of the third order is included to take into account the Kerr effect as follows:

$$
\vec{J}_{\text {Kerr }}=\epsilon_{0} \chi_{3} \frac{\partial\left(|\vec{E}|^{2} \vec{E}\right)}{\partial t},
$$

where the third-order susceptibility is $\chi_{3}=2 \times 10^{-22} \mathrm{~m}^{2} \mathrm{~V}^{-2}$ [71].

As typical intensities reached in our simulations are around or larger than $I=10^{17} \mathrm{~W} / \mathrm{m}^{2}$, we consider a complete Keldysh photoionization rate $w_{\text {pi }}$ [64], including multiphoton and tunneling ionization. The corresponding current is written as follows:

$$
\vec{J}_{\mathrm{pi}}=E_{g} \frac{w_{\mathrm{pi}} \vec{E}}{I} \frac{n_{a}-n_{e}}{n_{e}} .
$$


Here $E_{g}=9 \mathrm{eV}$ is the electron band gap in the absence of the electric field, $n_{a}=2 \times 10^{28} \mathrm{~m}^{-3}$ is the saturation density, $I=\frac{n}{2} \sqrt{\frac{\epsilon_{0}}{\mu_{0}}}|\vec{E}|^{2}$ is the intensity, and $n$ is the refractive index of unexcited fused silica with $n_{e}=0$. Note that applying six-photon ionization model instead of complete Keldysh ionization rate leads to an overestimation of ionization rate up to three orders in magnitude in our intensity interval $[62,72,73]$.

Maxwell's equations are then coupled to the multiple rate equations to calculate conduction band electron density by considering both electron avalanche and photoionization [63]. The system of $i_{m}$ coupled equations is written as follows:

$$
\begin{aligned}
\frac{\partial n_{1}}{\partial t} & =\left(n_{a}-n_{e}\right) w_{\mathrm{pi}}-W_{1, p t} n_{1}+2 \bar{\alpha} n_{i}-\frac{n_{1}}{\tau_{t r}}, \\
\frac{\partial n_{2}}{\partial t} & =W_{1, p t} n_{1}-W_{1, p t} n_{2}-\frac{n_{2}}{\tau_{t r}}, \\
& \cdots \\
\frac{\partial n_{i}}{\partial t} & =W_{1, p t} n_{i-1}-\bar{\alpha} n_{i}-\frac{n_{i}}{\tau_{t r}},
\end{aligned}
$$

where $i_{m}$ is defined by the critical energy of the impact ionization and the corresponding one-photon energy, the total electron carrier density $n_{e}=\sum_{i=1}^{i_{m}} n_{i}, \bar{\alpha}$ is the avalanche parameter, and $W_{1, p t}$ is the mean value of the one-photon absorption probability for fused silica. Unlike the single-rate equation, the multiple rate equation takes into account the fact that only the electrons of $i_{m}$ population, which have sufficiently high energy, contribute to the avalanche ionization [63]. The number of discrete energy states is calculated as $i_{m}=$ $1+\left[\frac{\epsilon_{\text {crit }}}{\hbar \omega_{L}}\right]$, where the critical energy for impact ionization is $\epsilon_{\text {crit }}=\left(1+m^{*} / m_{e}\right)\left(E_{g}+\frac{e^{2} I}{4 m^{*} \omega_{L}^{2}}\right)$, the reduced electron mass is $m^{*}=0.5 m_{e}$, the laser pulsation is $\omega_{L}=2 \pi c / \lambda$, and the corresponding photon energy is $\hbar \omega_{L}$. The one-photon absorption probability is defined as $W_{1, p t}=\frac{\sigma}{\ln (2) \epsilon_{\text {crit }}} \frac{1}{i_{m-1}-1} I$, where the absorption cross section is described by the Drude formalism as $\sigma=\frac{e^{2}}{m^{*} v_{e}\left[1+\left(\omega_{L} / v_{e}\right)^{2}\right]}$ [70]. For the avalanche parameter, we use the asymptotic value $\bar{\alpha}=\left[\sqrt[i_{m}-1]{2}-1\right] W_{1, p t}$ defined in Ref. [63]. In most of calculations, we solve a system of $i_{m}=10$ equations adopting the corresponding one-photon absorption probability. The performed numerical calculations demonstrate, however, that the results are slightly dependent on these parameters. This invariance is due to the fact that photoionization rate is much more significant than the avalanche one for subpicosecond pulses $[68,72,74,75]$. The recombination effect is included with $\tau_{t r}=150 \mathrm{fs}$ [76].

In this article we analyze the temporal evolution of the electron density distribution of plasma generated by ultrashort laser pulse irradiation during 1 fs pulse by solving the system of Eqs. (1)-(5). Herein, the terms "nanogratings,", "nanoplanes," or "nanostructures" designate only free carrier density profile. The experimental evidence of the electron density influence on the formation of the final structure can be found in [14,58,77,78].

A comment should be made on the modeling of the impurities of different nature in fused silica. The role of the chemistry in laser-induced structural glass modification was addressed in many studies [52-57,79-82]. Several exper- imental works reported the presence of color centers [53,55], voids [57,80], and oxygen [52,81] in laser-ablated glasses and also self-trapped excitons [71,79], which influence energy transport and lattice defect formation [31,32]. It is also known that oxygen vacancies lead to the narrowing of the material band gap $[52,55,83,84]$. According to the experimental data, only 1 from $10^{4}$ defects survives after each femtosecond laser shot [85]. It means that several pulses and electron densities significantly higher than $10^{24} \mathrm{~m}^{-3}$ are needed to induce permanent modification pulse by pulse [86], otherwise the number of defects after the laser shot would be comparable to the number of natural defects in the bulk of fused silica. When the larger number of electrons is generated from valence band to conducting band, the laser-induced structural changes involve the formation of new defects [87].

For simplicity we consider here nanoregions with a reduced ionization potential to be inhomogeneities formed as a result of multiple laser interactions. The inhomogeneities are initially set to be spheres of 5-10 nm radius of the dielectric material. The electromagnetic field in these regions is modeled by resolving the same system of Eqs. (1)-(5) with a narrower electron band gap $E_{g}=5.2 \mathrm{eV}[49,71,79,88]$. As long as the size of the inhomogeneity is considerably smaller than the irradiation wavelength, the initial plasma generation follows the Rayleigh scattering distribution [62]. The dynamics of the femtosecond laser interaction with such inhomogeneities is qualitatively the same giving us the opportunity to simulate up to $a=10 \mathrm{~nm}$ nanospheres for $\lambda=800 \mathrm{~nm}[48,62]$. The laser-induced inhomogeneity concentration increases with the number of pulses due to nonlinear ionization [58]. Therefore, we investigate the resulting electron density distribution by laser irradiation of randomly distributed inhomogeneities with different initial concentration corresponding to the state of laser-induced fused silica after several pulses. In fact, such an approach allows us not only studying the pulse number effect on the nanograting characteristics but also modeling of initially slightly doped fused silica with corresponding concentrations of inhomogeneities [15,59,61].

The initial Gaussian electric field profile is considered as a focused beam source with the beam waist of $w_{0}=$ $3 \mu \mathrm{m}$ and the numerical aperture NA $=n \sin \left(\frac{\lambda}{\pi w_{0}}\right)<0.2$ for irradiation wavelength from $\lambda=400 \mathrm{~nm}$ up to $\lambda=1200 \mathrm{~nm}$ relatively not high, for which the paraxial approximation of the Gaussian beam's profile is still valid $[35,49]$. Pulse width at half maximum (FWHM) is varied from $\theta=80$ fs up to $\theta=240$ fs. For such ultrashort pulses, ionization takes place before thermal effects such as heat diffusion occur [89], thus our numerical model, neglecting the electron-lattice exchange, is able to describe the modification in dielectric during the pulse duration. Based on the ionization memory effect [58], we suppose that the physical origin of the nanograting selforganization is the result of the electrodynamic processes and electron plasma evolution which take place during the pulse duration. Recent thermoelastoplastic modeling of laser-matter interaction has shown that the final modification structure is imprinted into bulk glass already at subnanosecond time scale [36].

First, we report the results of 2D-FDTD modeling of the TM-polarized mode. Thus, all the resulting snapshots of the electric fields, intensity, and electron density are in the plane 


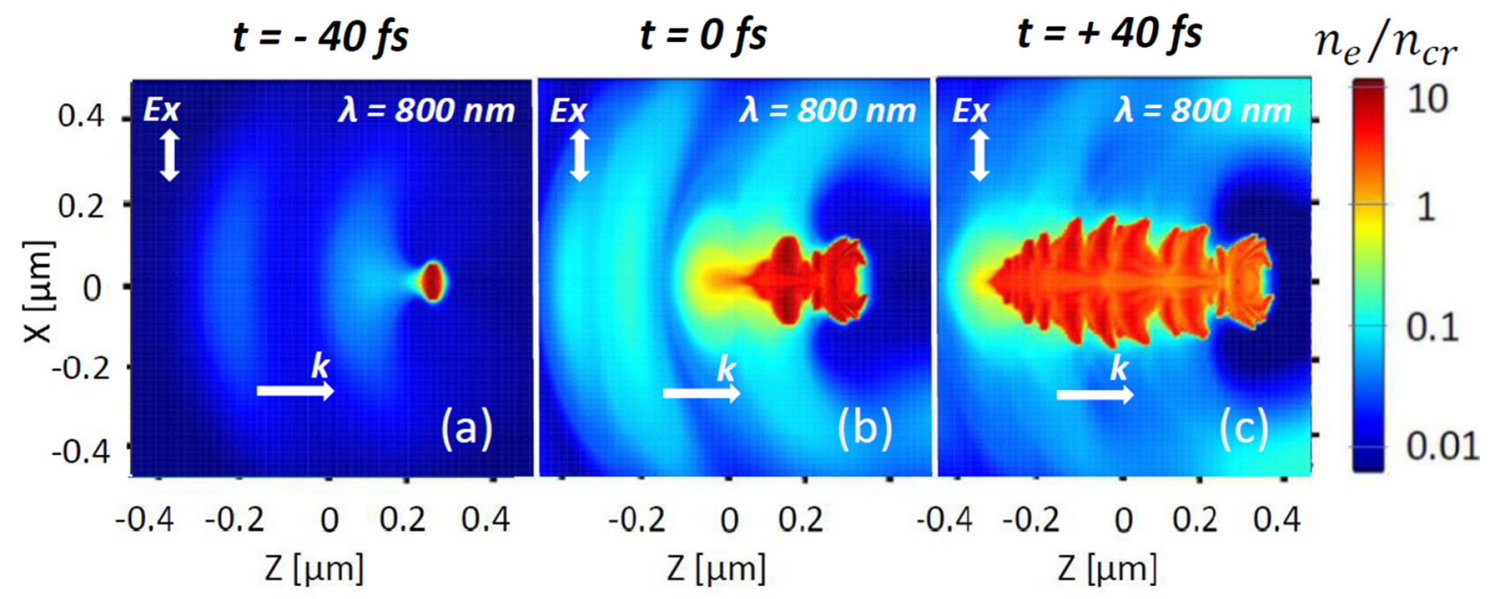

FIG. 1. Electron density snapshots are calculated by coupled 2D-FDTD (1)-(5) demonstrating the evolution from a single inhomogeneity of radius $a=5 \mathrm{~nm}$ localized in (0.3,0) $\mu \mathrm{m}$ to nanoplane taken at (a) $40 \mathrm{fs}$ before the pulse peak, (b) at the pulse peak, and (c) $40 \mathrm{fs}$ after the pulse peak. Laser irradiation conditions: pulse duration $\theta=120 \mathrm{fs}$ (FWHM), irradiation wavelength $\lambda=800 \mathrm{~nm}$, pulse energy $E=300 \mathrm{~nJ}$. Here and further, electron density is normalized to its critical value at $800 \mathrm{~nm} n_{\text {crit }}=1.7 \times 10^{27} \mathrm{~m}^{-3}$ and $\vec{k}$ is the incident laser wave vector.

$x O z$, where the focused beam is polarized along $x$ direction and $z$ is the propagation direction. Then, numerical results of 3D-FDTD modeling are presented and discussed.

\section{RESULTS AND DISCUSSION}

\section{A. Evolution from a single inhomogeneity to a nanoplane}

As an example, we start by considering femtosecond laser interaction with one spherical nanometric inhomogeneity of radius $a=5 \mathrm{~nm}$ in fused silica. Interestingly, even electron density profiles calculated around single inhomogeneity already reveal a remarkable plasma elongation backwards in the direction perpendicular to laser polarization in Fig. 1. Note that the process is very rapid and nonlinear. In addition, local field enhancement leads to sufficiently high intensities, so that the multiphoton ionization starts playing a crucial role changing the refractive index of the medium. Therefore, changes both in scattering and in absorption characteristics of the medium also take place.

In the near field of the nanoparticle, where $r \ll \lambda / 2 \pi n$, the intensity is strongly enhanced. One can thus expect that multiphoton ionization is also higher in these areas. The enhancement as well as the initial intensity distribution are different for inhomogeneities of different nature. For voids, or for inhomogeneities with an enhanced ionization cross section [48], the scattering is weaker than for metallic nanoparticles or high-density plasma nanospheres with reduced ionization cross section. Based on the parameters of the numerical model described in the previous part, we localize high-density plasma responsible for further elongation and formation of the nanograting. However, it is not the case for voids. To initiate the localized plasma generation in voids, we have to enhance the scattering changing the electron collision frequency parameter to $v_{e}=10^{14} \mathrm{~s}^{-1}$ previously used by [48]. The results of numerical calculations with a single void and with a high-density inhomogeneity demonstrate, however, that in spite of different nature of the inhomogeneities, initial intensity distribution and different parameters, the physics of the nanoplasma formation is qualitatively the same in both cases. It is related to the fact that after a thin plasma layer is generated in the near field of the initial nanosphere, further plasma generation is guided only by scattering from already organized plasma independent of the initial inhomogeneity nature. This means that the physics discussed in our article is applicable to any kind of initial inhomogeneities of the size rather smaller than the irradiation wavelength.

If the condition $r \ll \lambda / 2 \pi n$ is satisfied, the intensity distribution near nanometric laser-induced inhomogeneity is enhanced only in the direction of the electric field polarization. Figure 1(a) shows that plasma generated due to this enhancement has roughly an ellipsoid form. Interestingly, the size of the resulting stable ellipsoid depends only on the irradiation wavelength and optical parameters of the media. As the plasma dimension overcomes the laser wavelength range, we observe that the growth slows down and the backward scattering starts playing an essential role [Fig. 1(b)]. Such change in the scattering behavior [90] enables nanoplane elongation in the backward propagation direction [Fig. 1(c)].

In the far-field $r \gg \lambda / 2 \pi n$, the interference between the incident and the scattered wave leads to the organization of periodic standing spherical waves, with intensity proportional to $1 / r^{2}$ and with a period $\lambda / 2 n$ in the backward propagation direction [Fig. 1(a)]. This effect can be also observed for larger inhomogeneities consisting of quasimetallic plasma as in Fig. 1(b) and even for long nanoplanes acting as nanoantennas with reflectivity maxima at half-integer wavelength in the media [48]. Although the enhancement in the far-field backward propagation direction is rather weaker than in the near field and quickly decreases with $r$, Fig. 1(b) shows that it is possible to ionize the nearest enhanced regions under several tight focusing conditions by multiphoton absorption mechanism. As a result, the second nanoplasma structure is formed in the backward propagation direction. It affects the light propagation in a similar way. Figure 1(c) clearly demonstrates that such an incubation process results in the formation of a nanoplane elongated in the backward propagation direction perpendicular to the laser polarization. As the nanoplane is already formed, the process is now 


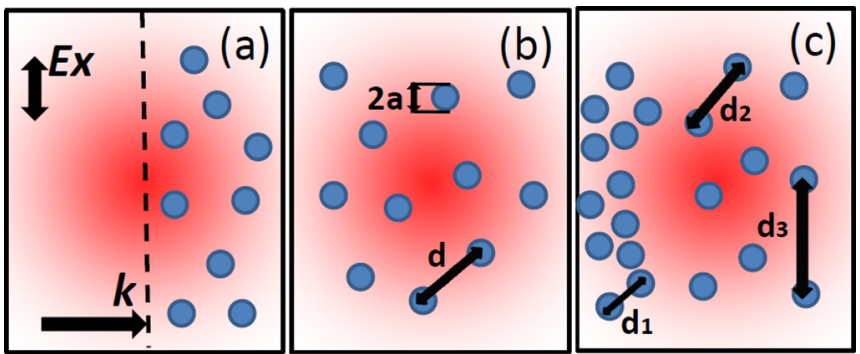

FIG. 2. Schematic representation of three possibilities of localization of laser-induced inhomogeneities before irradiation by femtosecond laser focused beam: (a) with interface between modified and unmodified regions, (b) with homogeneous concentration, and (c) with variable concentration $\left(d_{1}<d_{2}<d_{3}\right)$. $d$ is the average distance between the centers of the nanospheres and $a$ is the nanosphere radius.

guided mostly by the near-field intensity enhancement at the tip of the nanoplasma [2], leading to further elongation in the direction perpendicular to laser polarization. The length of the resulting nanoplasmas depends on the pulse duration and on the laser pulse energy, however, the final width in the polarization direction is independent of these parameters. High-fidelity visualization in fused silica confirms experimentally the evolution mechanism from a single inhomogeneity to nanocrack [7].

\section{B. Nanograting organization from randomly distributed inhomogeneities}

In the previous section we have considered femtosecond laser-matter interaction with one nanosphere localized in fused silica. Now, attention is focused on a system of randomly distributed inhomogeneities. By using previously proposed idea of the pulse to pulse memory [58], we consider several distributions of the laser-generated inhomogeneities, as shown in Fig. 2. First, there are regions modified and unmodified by the previous laser pulse with an evident interface between them as it was experimentally reported in [7,9]. Second, the inhomogeneities can be uniformly distributed. And finally and more realistically, there is a smooth concentration profile.

\section{Sharp interface}

Let us consider the first case. To analyze how the nanoplanes evolve in the presence of a sharp interface between concentration profiles, we divide our calculation volume into two regions: unmodified, without any initial inhomogeneities, and modified, with randomly distributed inhomogeneities. Electron density snapshots taken during the pulse duration reveal the temporal evolution of nanoplanes (Fig. 3). Similar behavior was reported in experiments [7,9]. Under tight focusing conditions, interference between the incident and scattered waves and incubation process induced by multiphoton ionization lead to the organization of first nanoplanes from the laser-induced interface. If there are few laser-induced inhomogeneities, each nanoplane grows strictly from its seed. Figure 3(a) shows, however, that several periodic intensity patterns are obtained in the region free of inhomogeneities near the interface if the concentration of inhomogeneities is increased. Nanoplasmas start following these intensity enhancements, elongating perpendicular to the laser polarization with a common period of $\lambda / n$ in Figs. 3(b) and 3(c). Furthermore, we refer to this kind of nanogratings as low-frequency nanogratings (LFNGs). Moreover, in the region with initially localized inhomogeneities, corresponding to the second configuration in our numerical investigation, we observe structures with the periodicity twice smaller, high-frequency nanogratings (HFNGs).

To demonstrate the effect of the irradiation wavelength on the periodicity of LFNGs and HFNGs, Fig. 4 shows electron density profiles calculated for three laser wavelengths frequently used in the experiments with femtosecond lasers $[3,4,7-9,32,91]$. One can see that the periodicity of the self-organized nanoplanes is proportional to $\lambda$, in agreement
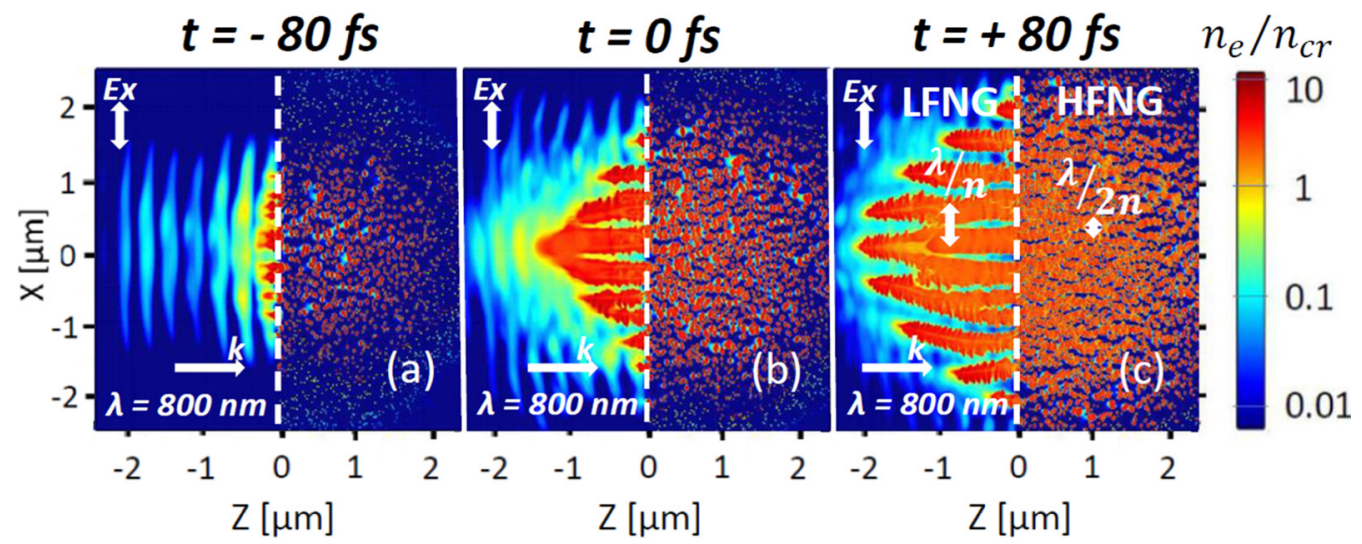

FIG. 3. Electron density snapshots are calculated by coupled 2D-FDTD (1)-(5) revealing the evolution of nanogratings (LFNGs and HFNGs) (a) $80 \mathrm{fs}$ before the pulse peak, (b) at the pulse peak, and (c) $80 \mathrm{fs}$ after the pulse peak. Laser irradiation conditions: pulse duration $\theta=240 \mathrm{fs}$ (FWHM), irradiation wavelength $\lambda=800 \mathrm{~nm}$, and pulse energy $E=500 \mathrm{~nJ}$. The line separates the region with initially localized nanospheres (modified) and the region, initially free from any inhomogeneities (unmodified). The schematic representation of the initial distribution of inhomogeneities is shown in Fig. 2(a). The concentration of the initial inhomogeneities in the right part of the volume is $C_{i}=1 \%$. 

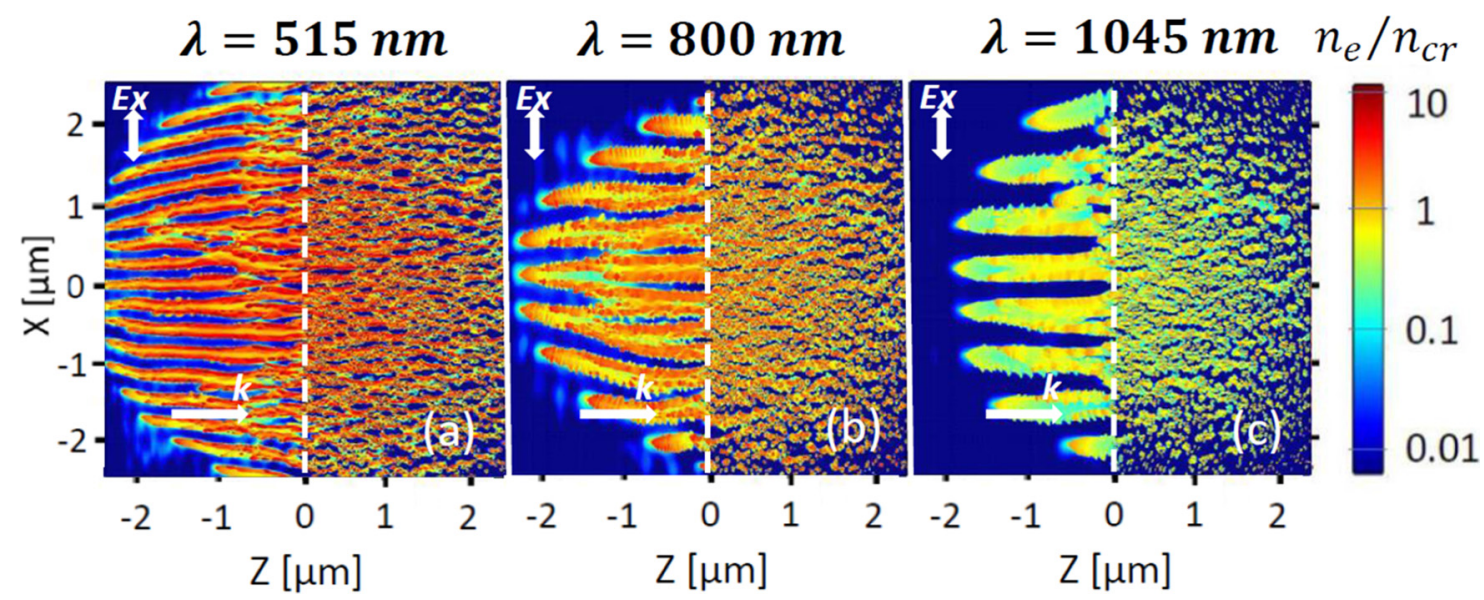

FIG. 4. Electron density snapshots are calculated by coupled 2D-FDTD (1)-(5) suggesting that the nanograting period is proportional to the irradiation wavelength: (a) $\lambda=515 \mathrm{~nm}$, (b) $\lambda=800 \mathrm{~nm}$, and (c) $\lambda=1045 \mathrm{~nm}$. Here electron density is normalized to its critical value at $800 \mathrm{~nm} n_{\text {crit }}=1.7 \times 10^{27} \mathrm{~m}^{-3}$. Laser irradiation conditions: pulse duration $\theta=240 \mathrm{fs}$ (FWHM) and corresponding pulse energies (a) $E=400 \mathrm{~nJ}$, (b) $E=500 \mathrm{~nJ}$, and (c) $E=600 \mathrm{~nJ}$. Snapshots are taken $80 \mathrm{fs}$ after the pulse peak. The line separates the region with initially localized nanospheres (modified) and the region, initially free from any inhomogeneities (unmodified). The schematic representation of the initial distribution of inhomogeneities is shown in Fig. 2(a). The concentration of the initial inhomogeneities in the right part of the volume is fixed to $C_{i}=0.5 \%$ for all wavelengths (a)-(c). Note that for each wavelength, different corresponding parameters are used for multiple rate equation (5) and Keldysh photoionization rate $w_{\mathrm{pi}}$.

with several experimental observations [2,8]. Interestingly, the nanoplanes become thicker with the increasing irradiation wavelength. First, we explain the physical origin of LFNGs. Second, we will focus on HFNGs, the structures with smaller periodicity.

Periodic structures formed at a rough interface between two media is usually associated with an interference between the incident wave and the scattered waves [92]. This interference causes periodic energy deposition [93-95]. In addition, an interference of the incident wave with the excited surface plasmon polaritons also leads to an enhancement of surface periodic structure formation under the conditions required for the surface plasmons [96-100]. Moreover, both scenarios were used to explain VNG formation $[9,33,41]$. Here, to elucidate the mechanism of the periodic organization of LFNGs, we study the role of each nonlinear current from the system of equations (1). The performed calculations demonstrate that the nanoplanes are formed even for $J_{\text {Kerr }}=0, J_{p i}=0$, and $J_{D z}=0$. The last condition means that we neglect the electron oscillation along the axis $z$. In this case the fields $E_{x}$ and $E_{z}$ can be directly separated if the intensity is given as $I=\frac{n}{2} \sqrt{\frac{\epsilon_{0}}{\mu_{0}}} E_{x}{ }^{2}$. Hence, electron density changes only due to the field component $E_{x}$. The periodicity of these nanoplanes is independent of the collision frequency of excited fused silica $v_{e}$ and periodic intensity patterns appear even for the case of nonexcited surface plasmons $\operatorname{Re}(\epsilon)>-n^{2}$ [97,99,100], where $\epsilon$ is dielectric permittivity defined by the Drude formalism. This fact suggests that the organization of LFNGs here is due to interface roughness. Furthermore, several phenomena contribute to the periodic nanoplane evolution: excitation of surface plasmon wave, scattering from the already organized elongated nanoplasmas, interference between the scattered waves from several laser-induced inhomogeneities, and backward scattering from a system of randomly distributed inhomogeneities.

\section{Smooth concentration profile}

Now, let us turn to a more general case of a smooth concentration profile, distributing randomly inhomogeneities in the entire numerical volume. Figure 5 reveals that nanograting formation process depends on the inhomogeneity concentration in this case. To describe the influence of the inhomogeneities on the nanoplane periodicity, we propose to define the average distance between two initial nanospheres as $d=1 / \sqrt{C_{i}}$, where $C_{i}=N / S$ is the concentration of inhomogeneities, $N$ is the number of the inhomogeneities in the laser-induced area $S$. The interference between multiple scattered waves from randomly distributed inhomogeneities strongly depends on this parameter. For widely separated nanospheres, the interference between the incident and the scattered fields dominates over all other plasmonic modes [101]. If particle dimensions are much smaller than the wavelength, the interference effect is negligible but interparticle coupling becomes significant, enabling high local concentration of electromagnetic energy in the vicinity of the conductive contact of nearly touching nanoplasmas [102].

If there are very few nanospheres, so that $d>\lambda / n$ $\left(C_{i}<0.05 \%\right)$, each inhomogeneity acts as a seed for one nanoplasma and they are elongated randomly in the grid. For higher concentration and if $\lambda / 2 n<d<\lambda / n(0.05 \%<$ $\left.C_{i}<0.5 \%\right)$ as in the case of pure dielectric with the seeds at its interface, the nanoplasmas elongate perpendicular to the laser polarization direction from the laser-modified inhomogeneities and the dominant period is close to $\lambda / n$ due to the described interference mechanism leading to the organization of LFNGs [Figs. 5(a) and 5(d)]. However, the mechanism of the organization changes dramatically for larger concentration of inhomogeneities. This effect is due to the mutual enhancement induced by multiple scattering from nanoplasmas that becomes dominant over the interference between the incident and the scattered fields. 


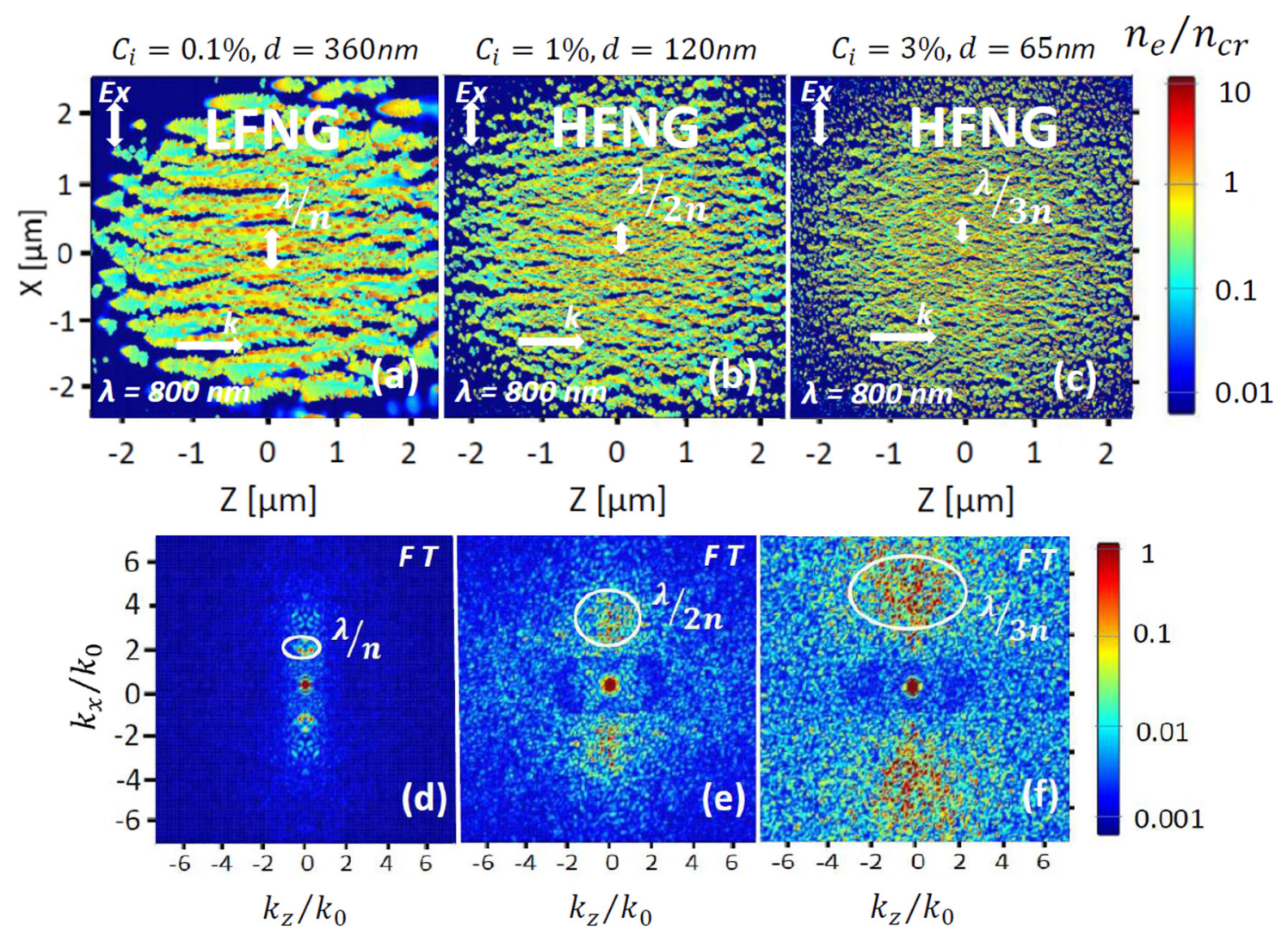

FIG. 5. (a)-(c) Electron density snapshots are calculated by coupled 2D-FDTD (1)-(5) at the end of the pulse duration $\theta=240 \mathrm{fs}$ (FWHM) for different initial concentration of randomly distributed inhomogeneities and (d)-(f) Fourier transform (FT) of the images: (a) and (d) $C_{i}=0.1 \%$, (b) and (e) $C_{i}=1 \%$, and (c) and (f) $C_{i}=3 \%$. The wave numbers $k_{z}$ and $k_{x}$ are normalized to the norm of the incident wave number $k_{0}=2 \pi / \lambda$. The pulse energy is fixed to $E=500 \mathrm{~nJ}$. The irradiation wavelength is $800 \mathrm{~nm}$. The schematic representation of the initial distribution of inhomogeneities is shown in Fig. 2(b).

Electron density snapshots demonstrate the temporal evolution of HFNGs from randomly distributed initial inhomogeneities in Fig. 2. The organization of HFNGs is completely different from LFNG formation. Even at the pulse peak no periodicity can be revealed yet. Nanoplasmas develop from random inhomogeneities into well-ordered patterns consequently, controlled by strong local field enhancement. From the Fourier transform of the electron density snapshot taken at the end of the pulse [Fig. 5(e)], one can see that the dominant period is close to $\lambda / 2 n$. The periodicity does not depend on the laser energy density which is the highest in the center and decreases towards the edge of the focal region as a Gaussian distribution. Note that several phenomena contribute to the observed period reduction such as intensity enhancement due to multiple scattering from the nanospheres, intensity enhancement between two organized nanoplasmas [19,41,103], and half-wavelength cavity feedback [33]. If we continue to increase inhomogeneity concentration, we observe that nanoplasmas tend to merge together. Figures 5(c) and 5(f) show that we are still able to reproduce self-organized nanogratings separated by $\lambda / 3 n$. At larger concentration, there is no more dominant characteristic period. This fact is due to a very short average distance between the initial nanospheres, as $d \approx 50-100 \mathrm{~nm}\left(C_{i} \approx 5 \%\right)$. The plasma is organized by femtosecond laser irradiation of such inhomogeneities and occupies all the gaps between inhomogeneities. Thus, VNGs spaced by less than $\lambda / 4 n$ are out of the scope of the present modeling.

To identify the physical phenomenon responsible for the organization of HFNGs, as in the previous case, we study the contributions of nonlinear currents in nanograting formation from the system of equations (1). First, we check that $J_{\text {Kerr }}$ and $J_{\text {pi }}$ do not influence the physical process. Second, we perform calculations with $J_{D z}=0$ to check whether the role of electron oscillations along the axis $z$ is negligible. In this case we observe no periodic organization. The electron density snapshots in Fig. 6 reveal that even for high concentration, the inhomogeneities do not interact creating nanoplanes elongated perpendicular to the laser polarization. We underline that the same procedure of switching off the $J_{D z}$ component in the case of LFNGs does not destroy the self-organization process. It means that different physical processes are responsible for creating LFNGs and HFNGs. Numerical calculations show that the component $\frac{e^{2} n_{e}}{m_{e}} E_{z}$ from Eq. (2) results in organization of the structures with a period smaller than $\lambda / n$. Hence, the physical origin of HFNGs is the interference of multiple scattered waves of the orthogonal field $E_{z}$ reinforced by growing electron concentration $n_{e}$. Interestingly, no HFNGs are formed in the case of the ideal plasma $v_{e}=0$. Therefore, the quasimetallic properties of plasma and the absorption play an important role in HFNG organization. 


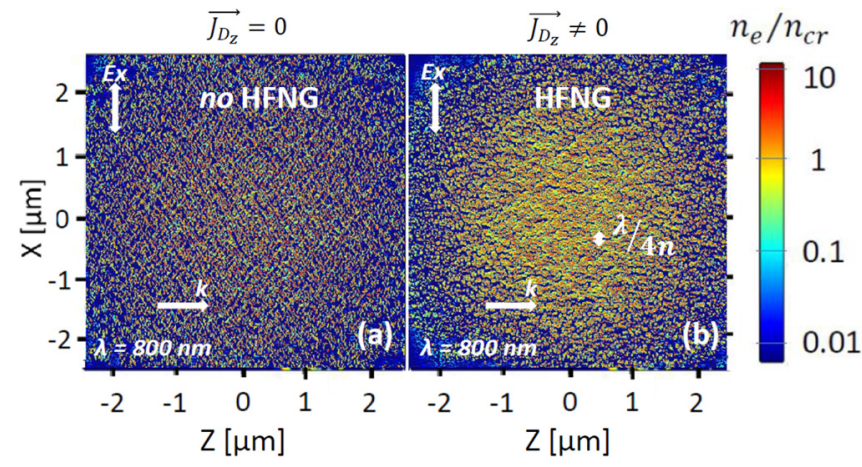

FIG. 6. Electron density snapshots are obtained by coupled 2DFDTD (1)-(5) assuming (a) $J_{D z}=0$ and (b) $J_{D z} \neq 0$ at the end of the pulse duration $\theta=240 \mathrm{fs}$ (FWHM), laser wavelength of $800 \mathrm{~nm}$ and pulse energy of $500 \mathrm{~nJ}$ with initial concentration of inhomogeneities $C_{i}=9 \%$. The schematic representation of the initial distribution of inhomogeneities is shown in Fig. 2(b).

\section{Smooth gradient profile}

Finally, to demonstrate the influence of concentration on the nanograting period, we consider the third case with a smooth gradient profile. The results shown in Fig. 7 are obtained by varying the average distance between initial nanospheres from 50 to $500 \mathrm{~nm}$ in space along the axis $z$. Electron density reveals changes in the nanograting periodicity and thickness corresponding to initial local concentration of inhomogeneities. LFNGs with period of $\lambda / n$ do not have initially the interface with modified region and grow in the backward propagation direction. Therefore, the resulting structure formation is not related to the surface wave excitation as it was suggested

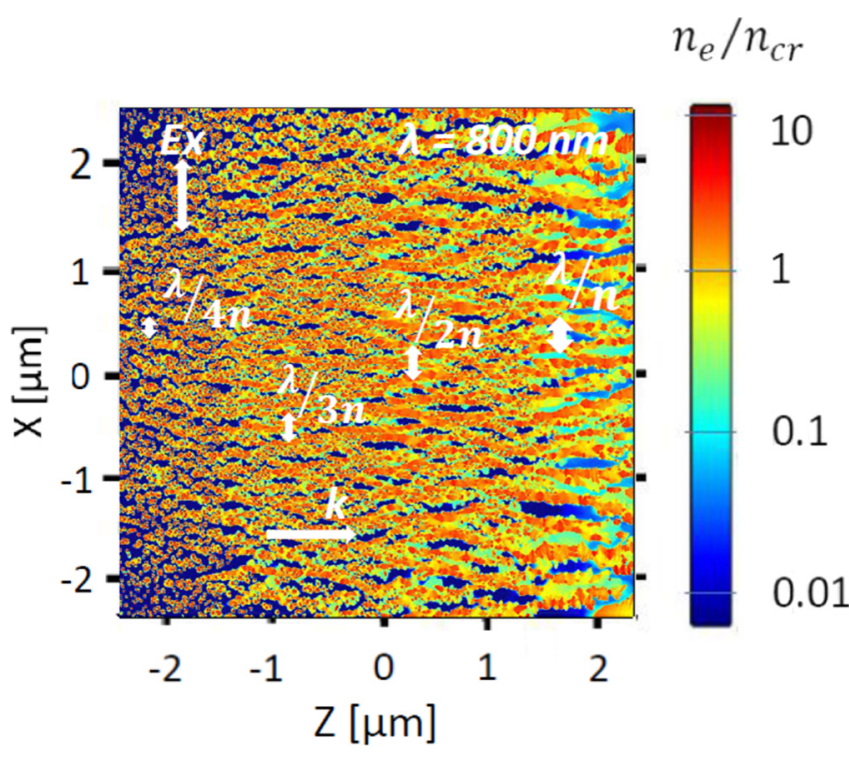

FIG. 7. Electron density snapshot is obtained by coupled 2DFDTD (1)-(5) taken 80 fs after the peak of the pulse duration $\theta=240 \mathrm{fs}$ (FWHM), laser wavelength of $800 \mathrm{~nm}$, and pulse energy of $500 \mathrm{~nJ}$ with initial variable concentration of inhomogeneities decreasing linearly in the $z$ direction from $C_{i}=5 \%$ to $C_{i}=0.05 \%$. The schematic representation of the initial distribution of inhomogeneities is shown in Fig. 2(c). in $[9,41]$. Instead, it is an entirely volume effect as it was previously underlined by Buschlinger et al. [48]. If we inverse the gradient of the concentration, we see that nanostructures correspond again to the initial concentrations. Thus, the physical origin of subwavelength self-organized structures is connected to the stationary wave induced either by the interference of the incident and inhomogeneity-scattered waves in the case of LFNGs, or by an interference of several scattered waves from multiple inhomogeneities in the case of HFNGs. By increasing the concentration, we change the scattering behavior. As a result, nanoplanes with different periodicity are obtained depending on the local inhomogeneity concentration.

\section{Comparison with experimental literature data}

In the previous section we have examined ultrashort laser irradiation of fused silica with initial randomly distributed inhomogeneities. This section presents a more detailed comparison with the available experimental results.

Figure 8 shows the calculated electron density distribution for various pulse energy. One can see that calculation results clearly confirm the previously observed three regimes of structural modifications [4]. For laser pulse with energies smaller than $0.1 \mu \mathrm{J}$, the modification is smooth and is characterized by a uniform positive change in the material refractive index, as soon as the electron densities do not exceed the critical value [i, Fig. 8(a)]. Thus, the scattering from the laser-induced inhomogeneities is rather weak to initiate the growth of planar nanoplasmas. By increasing the pulse energy, we enable their elongation perpendicular to the laser polarization [ii, Fig. 8(b)]. In addition, by varying energy from 0.2 to $1 \mu \mathrm{J}$, no changes in nanogratings period are observed, as experimentally reported in $[2,11,91,104]$. However, beyond the energy of $1 \mu \mathrm{J}$, high densities above the critical value are created in the center of tightly focused region, erasing the nanogratings at their initial state. Therefore, a complex disrupted region is interconnected with nanoplanes in the top portion of the laser-induced region [iii, Fig. 8(c)].

To examine the role of temporal pulse width, we vary pulse duration from $\theta=80$ fs up to $\theta=240$ fs. We observe that the nanogratings are self-organized with the same characteristic period for each of these pulse durations. These results also agree with the following experiments $[1,2,4,10,104]$. Note that the nanogratings with a characteristic period of $\lambda / 2 n$ have been recently revealed even for pulse duration as long as 8 ps [89].

In what follows, we investigate periodic organization of nanostructures from randomly distributed inhomogeneities. Electron density distributions calculated by coupled FDTD (1)-(5) have strong similarities with the nanogratings observed in most experiments. A good agreement between the periods obtained numerically and experimentally [2-5] is achieved. It is worth noting that the proposed mechanism of nanograting self-organization also explains possible coexistence of several characteristic periods [11,105] as well as the nanostructures separated by subwavelength periods shorter (around $\lambda / 3 n$ ) [1,8,18,27,59] at higher number of pulses and larger (around $\lambda / n)[9,10,106,107]$ than $\lambda / 2 n$. According to numerical calculations, a uniform concentration profile of inhomogeneities is the most probable in the experiments, probably because of the strong arrangement 

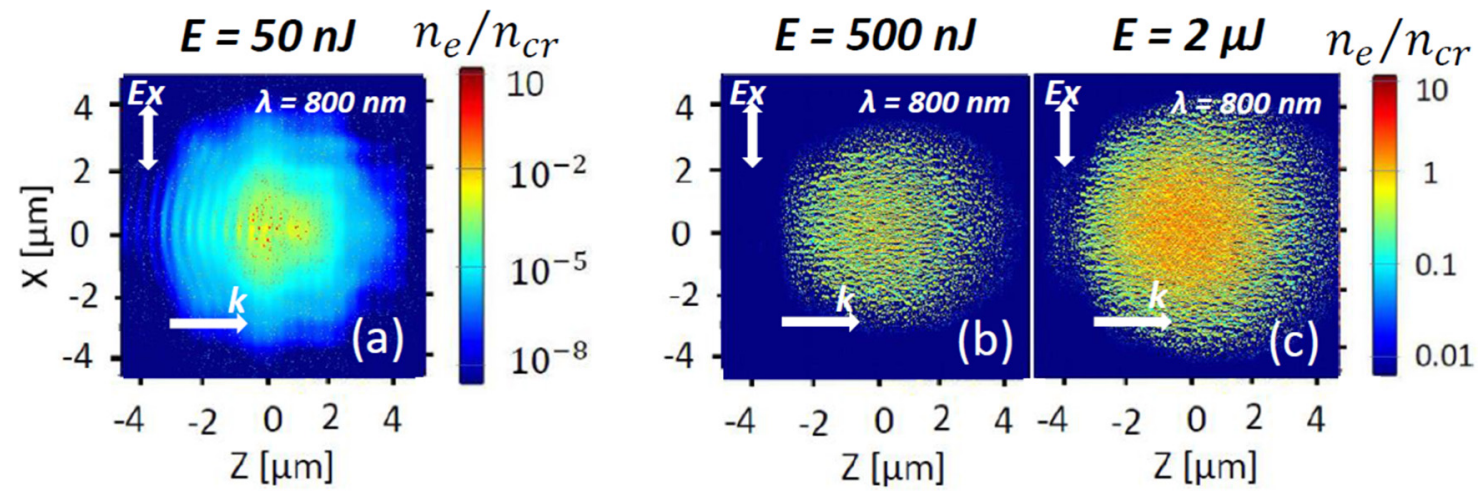

FIG. 8. Electron density snapshots are obtained by coupled 2D-FDTD (1)-(5) taken at the end of the pulse duration $\theta=240$ fs (FWHM) and laser wavelength of $800 \mathrm{~nm}$ with laser beam energy: (a) $E=50 \mathrm{~nJ}$ (smooth modification), (b) $E=500 \mathrm{~nJ}$ (birefringent modification), and (c) $E=2 \mu \mathrm{J}$ (disrupted modification). Electron density is normalized to its critical value at $800 \mathrm{~nm} n_{\text {crit }}=1.7 \times 10^{27} \mathrm{~m}^{-3}$. Note that in (a) the scale is different than in (b) and in (c). The schematic representation of the initial distribution of inhomogeneities is shown in Fig. 2(b). The concentration of the initial inhomogeneities is $C_{i}=1 \%$. Note that different number of rate equations (5) are solved for different amplitudes of the electric field: (a) $i_{m}=9$, (b) $i_{m}=10$, and (c) $i_{m}=21$.

due to high number of pulses. Moreover, we underline the crucial role of laser-induced inhomogeneities in nanograting self-organization and the possibility of regulating the period of the nanostructures changing the inhomogeneity concentration as it has been recently evidenced experimentally either by doping fused silica $[15,59]$ or by varying the applied number of pulses [10,13,19,107].

Interestingly, similar experimental results of nanograting period dependence on laser pulse number were obtained for $\lambda=515 \mathrm{~nm}$ [8] and for $\lambda=1550 \mathrm{~nm}$ [10]. In fact, these experimental findings correlate fairly well with the concentration of inhomogeneities in numerical modeling, as Fig. 9 shows. Based on the mechanism of nonlinear ionization memory from pulse to pulse [58], new inhomogeneities are organized pulse by pulse. Hence the concentration of inhomogeneities is proportional to the number of pulses. Such assumption allows us to explain the phenomenon of the decreasing nanograting

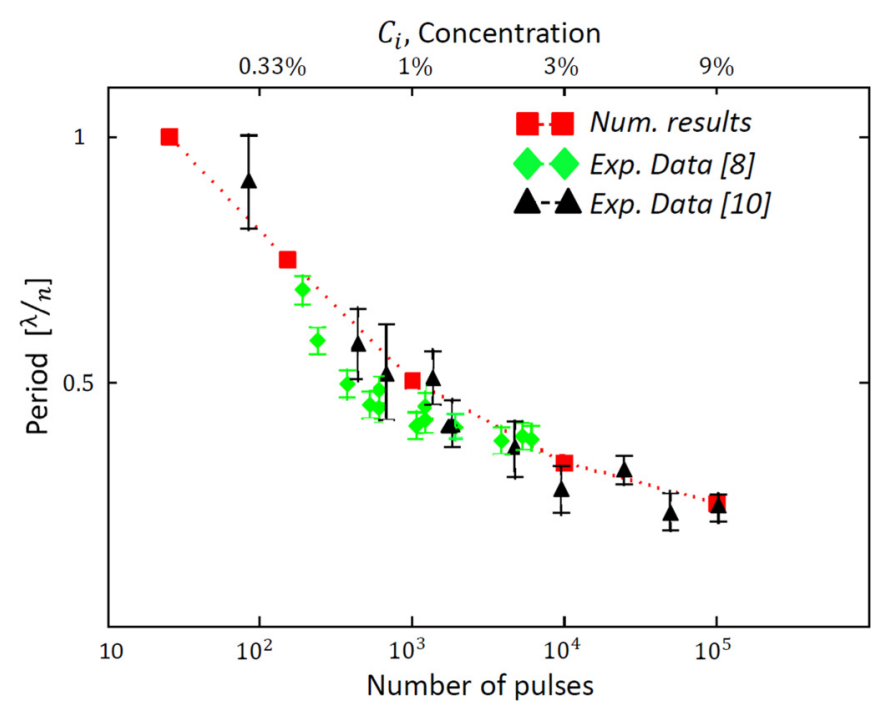

FIG. 9. Period dependence on the number of pulses from experimental data $[8,10]$ comparing with the dependence on the concentration of inhomogeneities from numerical modeling. period in terms of the decreasing average distance between the laser-induced inhomogeneities. The period depends strongly on the concentration and continuously decreases depending on different ways of scattering from the multiple nanoplasmas. We note that the exact values of number of pulses are correlated with the corresponding inhomogeneity concentrations without any straightforward equivalence. Thus, if the applied number of pulses remains low, the periodicity sharply decreases. At high number of pulses, a smoother transition emerges. The same tendency has been obtained for low and high inhomogeneity concentrations.

In our calculations, each nanoplane consists of numerous high density laser-induced plasmas initiated from randomly distributed initial inhomogeneities and "frozen" at different steps of their evolution. Thus, there are two types of nanoplasmas: (i) those that are significantly elongated up to several hundreds of nanometers perpendicular to the laser polarization, and (ii) the other ones with a diameter of 10-20 nm. Similar structures were observed in $[5,10,78]$.

Finally, the results of a series of complete 3D-FDTD simulations are presented in Fig. 10. Electron density snapshot taken in the plane $x O y$ perpendicular to the laser wave propagation presented in Fig. 10(b) reveals the orientation of the self-organized nanogratings strictly perpendicular to the laser polarization. Figure 10(d) demonstrates that by changing the polarization of the electric field, we rotate the nanoplanes in space. Again, we obtain the nanograting orientation perpendicular to the laser polarization in agreement with the experimental observations $[2,11,17]$. The results in $x O z$ plane by 2D-FDTD are shown to be consistent with the results obtained by 3D-FDTD. By switching off the currents $J_{D z}$ or $J_{D y}$, we find that the self-organization process does not occur anymore in both cases. This fact proves that the structures are formed by an interference of the multiple scattered waves of the orthogonal fields $E_{z}$ and $E_{y}$. This implies that the role of the electron oscillations along $z$ and $y$ axes is not negligible. Therefore, the structures correspond to the HFNG type. The periodicity of the structures is close to the half of the laser wavelength in glass. We note that the same periodicity is revealed by our 2D-FDTD calculations with the same initial 


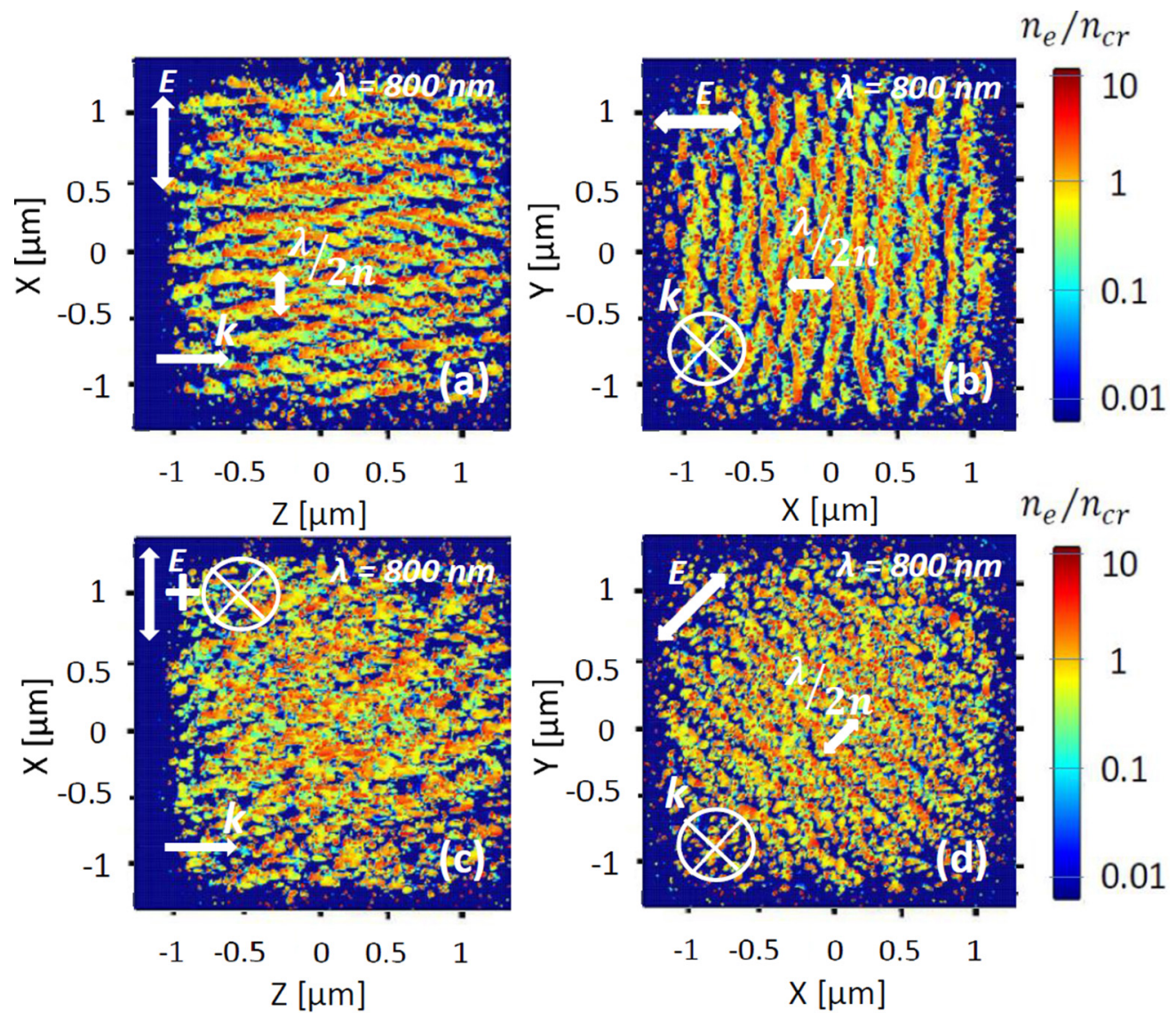

FIG. 10. Electron density snapshots are obtained by coupled 3D-FDTD (1)-(5) taken at the end of the pulse duration $\theta=120 \mathrm{fs}$ (FWHM) with laser beam energy $E=300 \mathrm{~nJ}$ and irradiation wavelength of $800 \mathrm{~nm}$ for different electric field polarizations: (a) and (b) electric field is along $O x$, and (c) and (d) electric field lies in $x O y$ and has $\pi / 4$ angle with the axe $O x$. Electron density is normalized to its critical value at $800 \mathrm{~nm} n_{\text {crit }}=1.7 \times 10^{27} \mathrm{~m}^{-3}$. The schematic representation of the initial distribution of inhomogeneities is shown in Fig. 2(b). The concentration of the initial inhomogeneities is $C_{i}=1 \%$.

inhomogeneity concentration of smooth distribution profile. We underline that the orientation of the HFNG structures is always perpendicular to the laser polarization.

The presented model does not describe all sets of complex thermomechanical and chemical processes taking place between two pulses in fused silica during VNG formation. Nevertheless, these simplified calculation results help to shed new light at the role of laser-induced inhomogeneities, interference between the incident and inhomogeneityscattered waves, multiple scattering, local field enhancement resulting in plasma elongation, and nanograting selforganization in direction perpendicular to the laser irradiation. In particular, the model is able to explain the previously observed period to be close to $\lambda / 2 n$. The development of a more complete model is underway and is out of the scope of the present article.

\section{CONCLUSION}

We have numerically investigated the mechanisms of VNG formation under femtosecond laser irradiation of fused silica with randomly distributed nanometric inhomogeneities by solving nonlinear Maxwell's equation coupled with multiple rate electron density equation taking into account both avalanche and complex Keldysh photoionization. Our numeri- cal model is capable to reproduce three regimes of modification in fused silica: (i) smooth modification, (ii) birefringent modification enabling the nanograting self-organization, and (iii) complex disruptive modification at higher laser pulse energies.

By analyzing the calculated electron density distribution, we have revealed and explained the evolution from small spherical nanometric inhomogeneities to periodical nanoplanes. Three cases have been considered: (i) with a sharp interface between modified and unmodified regions, (ii) with a constant concentration, and (iii) with a smooth concentration profile. In all these cases, periodic nanostructure formation has been observed. The obtained results have shown that the period of nanoplanes growing perpendicularly to the laser polarization depends strongly on the irradiation wavelength. These facts agree fairly well with previous experimental observations. Furthermore, 3D-FDTD modeling has shown that nanostructures can be rotated in space by changing the electric field polarization.

The nanograting formation is attributed to an interplay of several physical processes, such as an interference between the incident and the scattered waves, multiple scatterings, local field enhancement, and accumulation processes driven by multiphoton ionization. More particularly, we have underlined two different regimes of scattering responsible for 
the formation of the nanogratings with different periodicity. The first one is due to the interference between the incident wave and the inhomogeneity-scattered waves resulting into the organization of nanoplanes with a characteristic period of the laser irradiation wavelength in glass (LFNGs). Particularly, such phenomenon takes place in the presence of a sharp interface. The second one, for a smooth concentration profile, is due to the interference in multiple scattering from nearly touching inhomogeneities induced by the electric fields orthogonal to the laser polarization which leads to the formation of high-frequency nanogratings (HFNGs).

An explanation of the pulse number effect on the nanograting periodicity is furthermore proposed based on the mechanism of the nonlinear ionization memory and correlation between the concentration of inhomogeneities and the number of pulses. As the concentration is related to the separation distance between laser-induced nanospheres, the periodicity decreases with the increasing number of pulses due to different regimes of multiple scattering on nanoscale inhomogeneities.

Finally, the performed numerical modeling shows that laser-induced inhomogeneities play a crucial role in VNG formation. The nanometric inhomogeneities are not only the seeds and the initial reason for periodic nanograting selforganization [48] but also the attributes guiding the whole process. Without these seeds, it is impossible to explain nanoplane period and their thickness. Thus, a control over the nanostructure properties is probably possible by slightly doping fused silica. The performed study helps to better understand the phenomena involved in volume nanograting development, which is crucial for the development of a wide range of applications in femtosecond laser material processing, particularly, nanofluidic channels for biomedicine and DNA molecular analysis, optical data storage devices, and computer-generated holograms for informatics [20-26].

\section{ACKNOWLEDGMENTS}

This work was supported by the NANODIELEC project, LABEX MANUTECH SISE (ANR-10-LABEX-0075) of Université de Lyon, within the program "Investissements d'Avenir" (ANR-11-IDEX-0007) operated by the French National Research Agency (ANR).
[1] Y. Shimotsuma, P. G. Kazansky, J. Qiu, and K. Hirao, Phys. Rev. Lett. 91, 247405 (2003).

[2] V. R. Bhardwaj, E. Simova, P. P. Rajeev, C. Hnatovsky, R. S. Taylor, D. M. Rayner, and P. B. Corkum, Phys. Rev. Lett. 96, 057404 (2006).

[3] P. G. Kazansky, W. Yang, E. Bricchi, J. Bovatsek, A. Arai, Y. Shimotsuma, K. Miura, and K. Hirao, Appl. Phys. Lett. 90, 151120 (2007).

[4] R. Taylor, C. Hnatovsky, and E. Simova, Laser Photon. Rev. 2, 26 (2008).

[5] S. Richter, A. Plech, M. Steinert, M. Heinrich, S. Döring, F. Zimmermann, U. Peschel, E. Kley, A. Tünnermann, and S. Nolte, Laser Photon. Rev. 6, 787 (2012).

[6] C. Mauclair, M. Zamfirescu, J. P. Colombier, G. Cheng, K. Mishchik, E. Audouard, and R. Stoian, Opt. Express 20, 12997 (2012).

[7] Y. Liao, J. Ni, L. Qiao, M. Huang, Y. Bellouard, K. Sugioka, and Y. Cheng, Optica 2, 329 (2015).

[8] S. Richter, M. Heinrich, S. Döring, A. Tünnermann, S. Nolte, and U. Peschel, J. Laser Appl. 24, 042008 (2012).

[9] M. Hörstmann-Jungemann, J. Gottmann, and D. Wortmann, J. Laser Micro/Nanoeng. 4, 135 (2009).

[10] F. Zimmermann, S. Richter, R. Buschlinger, S. Shukla, R. Heintzmann, U. Peschel, and S. Nolte, Optically Induced Nanostructures: Biomedical and Technical Applications (Walter de Gruyter, Berlin, 2015), pp. 93-115.

[11] F. Zhang, H. Zhang, G. Dong, and J. Qiu, J. Opt. Soc. Am. B 31, 860 (2014).

[12] Y. Li, V. A. Stoica, L. Endicott, G. Wang, H. Sun, K. P. Pipe, C. Uher, and R. Clarke, Appl. Phys. Lett. 99, 121903 (2011).

[13] M. S. Ahsan, Y. G. Kim, and M. S. Lee, J. Non-Cryst. Solids 357, 851 (2011).

[14] M. Lancry, R. Desmarchelier, F. Zimmermann, N. Guth, F. Brisset, S. Nolte, and B. Poumellec, Advanced Photonics
(Optical Society of America, Washington, DC, 2014), p. BW2D.2.

[15] M. Lancry, J. Canning, K. Cook, M. Heili, D. R. Neuville, and B. Poumellec, Opt. Mater. Express 6, 321 (2016).

[16] C. Hnatovsky, V. Shvedov, W. Krolikowski, and A. Rode, Phys. Rev. Lett. 106, 123901 (2011).

[17] Y. Dai, G. Wu, X. Lin, G. Ma, and J. Qiu, Opt. Express 20, 18072 (2012).

[18] S. Richter, M. Heinrich, S. Döring, A. Tünnermann, and S. Nolte, Appl. Phys. A 104, 503 (2011).

[19] Y. Liao, W. Pan, Y. Cui, L. Qiao, Y. Bellouard, K. Sugioka, and Y. Cheng, Opt. Lett. 40, 3623 (2015).

[20] Y. Shimotsuma, M. Sakakura, P. G. Kazansky, M. Beresna, J. Qiu, K. Miura, and K. Hirao, Adv. Mater. 22, 4039 (2010).

[21] M. Beresna, M. Gecevičius, and P. G. Kazansky, Opt. Mater. Express 1, 783 (2011).

[22] W. Cai, A. R. Libertun, and R. Piestun, Opt. Express 14, 3785 (2006).

[23] Y. Liao and Y. Cheng, Micromachines 5, 1106 (2014).

[24] Y. Liao, Y. Cheng, C. Liu, J. Song, F. He, Y. Shen, D. Chen, Z. Xu, Z. Fan, X. Wei, K. Sugioka, and K. Midorikawa, Lab Chip 13, 1626 (2013).

[25] C. Hnatovsky, R. Taylor, E. Simova, P. Rajeev, D. Rayner, V. Bhardwaj, and P. Corkum, Appl. Phys. A 84, 47 (2006).

[26] M. Beresna, M. Gecevičius, P. G. Kazansky, and T. Gertus, Appl. Phys. Lett. 98, 201101 (2011).

[27] F. Liang, J. Bouchard, S. Leang Chin, and R. Vallée, Appl. Phys. Lett. 107, 061903 (2015).

[28] F. Liang, Q. Sun, D. Gingras, R. Vallée, and S. L. Chin, Appl. Phys. Lett. 96, 101903 (2010).

[29] F. Liang, R. Vallée, D. Gingras, and S. L. Chin, Opt. Mater. Express 1, 1244 (2011).

[30] F. Liang, R. Vallée, and S. L. Chin, Opt. Express 20, 4389 (2012). 
[31] S. Richter, F. Jia, M. Heinrich, S. Döring, U. Peschel, A. Tünnermann, and S. Nolte, Opt. Lett. 37, 482 (2012).

[32] M. Beresna, M. Gecevičius, P. G. Kazansky, T. Taylor, and A. V. Kavokin, Appl. Phys. Lett. 101, 053120 (2012).

[33] R. Buividas, L. Rosa, R. Šliupas, T. Kudrius, G. Šlekys, V. Datsyuk, and S. Juodkazis, Nanotechnology 22, 055304 (2011).

[34] E. S. Efimenko, A. V. Kim, and M. Quiroga-Teixeiro, Phys. Plasmas 18, 032107 (2011).

[35] N. M. Bulgakova, V. P. Zhukov, and Y. P. Meshcheryakov, Appl. Phys. B 113, 437 (2013).

[36] N. M. Bulgakova, V. P. Zhukov, S. V. Sonina, and Y. P. Meshcheryakov, J. Appl. Phys. 118, 233108 (2015).

[37] R. L. Harzic, D. Dörr, D. Sauer, M. Neumeier, M. Epple, H. Zimmermann, and F. Stracke, Opt. Lett. 36, 229 (2011).

[38] A. P. Singh, A. Kapoor, K. Tripathi, and G. Kumar, Opt. Laser Technol. 34, 37 (2002).

[39] Y. Dong and P. Molian, Appl. Phys. Lett. 84, 10 (2004).

[40] V. S. Makin and R. S. Makin, Opt. Spectrosc. 115, 591 (2013).

[41] Y. Liao, J. Ni, L. Qiao, M. Huang, Y. Bellouard, K. Sugioka, and Y. Cheng, Proc. SPIE, Vol. 9350 (Optical Society of America, Washington, DC, 2015), p. 93500.

[42] B. Poumellec, M. Lancry, R. Desmarchelier, E. Hervé, F. Brisset, and J. Poulin, Opt. Mater. Express 3, 1586 (2013).

[43] L. Ramirez, M. Heinrich, S. Richter, F. Dreisow, R. Keil, A. Korovin, U. Peschel, S. Nolte, and A. Tünnermann, Appl. Phys. A 100, 1 (2010).

[44] N. Bulgakova and V. Zhukov, Lasers in Materials Science, Springer Series in Materials Science, Vol. 191 (Springer, Berlin, 2014), pp. 101-124.

[45] A. Couairon, L. Sudrie, M. Franco, B. Prade, and A. Mysyrowicz, Phys. Rev. B 71, 125435 (2005).

[46] I. M. Burakov, N. M. Bulgakova, R. Stoian, A. MermillodBlondin, E. Audouard, A. Rosenfeld, A. Husakou, and I. V. Hertel, J. Appl. Phys. 101, 043506 (2007).

[47] J. R. Gulley and W. M. Dennis, Phys. Rev. A 81, 033818 (2010).

[48] R. Buschlinger, S. Nolte, and U. Peschel, Phys. Rev. B 89, 184306 (2014).

[49] N. M. Bulgakova, V. P. Zhukov, Y. P. Meshcheryakov, L. Gemini, J. Brajer, D. Rostohar, and T. Mocek, J. Opt. Soc. Am. B 31, C8 (2014).

[50] K. I. Popov, C. McElcheran, K. Briggs, S. Mack, and L. Ramunno, Opt. Express 19, 271 (2010).

[51] H. Schmitz and V. Mezentsev, J. Opt. Soc. Am. B 29, 1208 (2012).

[52] T. Bakos, S. N. Rashkeev, and S. T. Pantelides, Phys. Rev. B 69, 195206 (2004).

[53] S. Guizard, P. Martin, G. Petite, P. D’Oliveira, and P. Meynadier, J. Phys.: Condens. Matter 8, 1281 (1996).

[54] X. Gao, G. Feng, L. Zhai, and Z. Shouhuan, Opt. Engineering. 53, 026101 (2014).

[55] L. Skuja, H. Hosono, and M. Hirano, Proc. SPIE, Vol. 4347 (Optical Society of America, Washington, DC, 2001), pp. $155-168$.

[56] A. Zoubir, C. Rivero, R. Grodsky, K. Richardson, M. Richardson, T. Cardinal, and M. Couzi, Phys. Rev. B 73, 224117 (2006).

[57] R. H. Doremus, J. Am. Ceram. Soc. 49, 461 (1966).
[58] P. P. Rajeev, M. Gertsvolf, E. Simova, C. Hnatovsky, R. S. Taylor, V. R. Bhardwaj, D. M. Rayner, and P. B. Corkum, Phys. Rev. Lett. 97, 253001 (2006).

[59] F. A. Umran, Y. Liao, M. M. Elias, K. Sugioka, R. Stoian, G. Cheng, and Y. Cheng, Opt. Express 21, 15259 (2013).

[60] M. Vangheluwe, F. Liang, Y. Petit, P. Hée, Y. Ledemi, S. Thomas, E. Fargin, T. Cardinal, Y. Messaddeq, L. Canioni, and R. Vallée, Opt. Lett. 39, 5491 (2014).

[61] M. Lancry, B. Poumellec, A. Chahid-Erraji, M. Beresna, and P. G. Kazansky, Opt. Mater. Express 1, 711 (2011).

[62] A. Rudenko, J.-P. Colombier, and T. Itina, Proceedings of the 36th Progress in Electromagnetics Research Conference (PIERS), Prague, 2015 (The Electromagnetics Academy, Cambridge, MA, 2015), pp. 1870-1876.

[63] B. Rethfeld, Phys. Rev. Lett. 92, 187401 (2004).

[64] L. V. Keldysh, Sov. Phys. JETP 20, 1307 (1965).

[65] J. A. Roden and S. D. Gedney, Microwave Optical. Technol. Lett. 27, 334 (2000).

[66] J. H. Greene and A. Taflove, Opt. Express 14, 8305 (2006).

[67] E. G. Gamaly and A. V. Rode, J. Opt. Soc. Am. B 31, C36 (2014).

[68] V. V. Temnov, K. Sokolowski-Tinten, P. Zhou, B. Rethfeld, V. E. Gruzdev, A.-A. El-Khamawy, and D. von der Linde, Proc. SPIE, Vol. 5448 (Optical Society of America, Washington, DC, 2004), pp. 1119-1126.

[69] N. Varkentina, O. Uteza, N. Sanner, B. Chimier, M. Sentis, and T. Itina, Proc. SPIE, Vol. 7920 (Optical Society of America, Washington, DC, 2011), p. 792003.

[70] L. Gallais, D.-B. Douti, M. Commandré, G. Batavičiūte, E. Pupka, M. Ščiuka, L. Smalakys, V. Sirutkaitis, and A. Melninkaitis, J. Appl. Phys. 117, 223103 (2015).

[71] P. Martin, S. Guizard, P. Daguzan, G. Petite, P. D'Oliveira, P. Meynadier, and M. Perdrix, Phys. Rev. B 55, 5799 (1997).

[72] A. Q. Wu, I. H. Chowdhury, and X. Xu, Phys. Rev. B 72, 085128 (2005).

[73] B. Chimier, O. Utéza, N. Sanner, M. Sentis, T. Itina, P. Lassonde, F. Légaré, F. Vidal, and J. C. Kieffer, Phys. Rev. B 84, 094104 (2011).

[74] B. Rethfeld, Phys. Rev. B 73, 035101 (2006).

[75] N. S. Shcheblanov, E. P. Silaeva, and T. E. Itina, Appl. Surf. Sci. 258, 9417 (2012).

[76] B. Rethfeld, O. Brenk, N. Medvedev, H. Krutsch, and D. Hoffmann, Appl. Phys. A 101, 19 (2010).

[77] Y. Bellouard, E. Barthel, A. A. Said, M. Dugan, and P. Bado, Opt. Express 16, 19520 (2008).

[78] S. P. Sharma, V. Oliveira, P. Herrero, and R. Vilar, J. Appl. Phys. 116, 053106 (2014).

[79] C. Itoh, K. Tanimura, and N. Itoh, J. Phys. C 21, 4693 (1988).

[80] M. Hasegawa, M. Saneyasu, M. Tabata, Z. Tang, Y. Nagai, T. Chiba, and Y. Ito, Nucl. Instrum. Methods Phys. Res. Sect. B 166-167, 431 (2000).

[81] L. Bressel, D. de Ligny, E. G. Gamaly, A. V. Rode, and S. Juodkazis, Opt. Mater. Express 1, 1150 (2011).

[82] J. Canning, M. Lancry, K. Cook, A. Weickman, F. Brisset, and B. Poumellec, Opt. Mater. Express 1, 998 (2011).

[83] L. Skuja, J. Non-Cryst. Solids 239, 16 (1998).

[84] S. A. Ansari, M. M. Khan, S. Kalathil, A. Nisar, J. Lee, and M. H. Cho, Nanoscale 5, 9238 (2013). 
[85] G. Petite, P. Daguzan, S. Guizard, and P. Martin, Nucl. Instrum. Methods Phys. Res. Sect. B 107, 97 (1996).

[86] D. G. Papazoglou and S. Tzortzakis, Opt. Mater. Express 1, 625 (2011)

[87] S. Sen and J. E. Dickinson, Phys. Rev. B 68, 214204 (2003).

[88] K. Tanimura, C. Itoh, and N. Itoh, J. Phys. C 21, 1869 (1988).

[89] C. Corbari, A. Champion, M. Gecevičius, M. Beresna, Y. Bellouard, and P. G. Kazansky, Opt. Express 21, 3946 (2013).

[90] X. Fan, W. Zheng, and D. J. Singh, Light: Sci. Appl. 3, e179 (2014).

[91] D. Wortmann, J. Gottmann, N. Brandt, and H. Horn-Solle, Opt. Express 16, 1517 (2008).

[92] J. E. Sipe, J. F. Young, J. S. Preston, and H. M. van Driel, Phys. Rev. B 27, 1141 (1983).

[93] T. J.-Y. Derrien, R. Torres, T. Sarnet, M. Sentis, and T. E. Itina, Appl. Surf. Sci. 258, 9487 (2012).

[94] J. Z. P. Skolski, G. R. B. E. Römer, J. V. Obona, V. Ocelik, A. J. Huis in 't Veld, and J. T. M. De Hosson, Phys. Rev. B 85, 075320 (2012).

[95] H. Zhang, J.-P. Colombier, C. Li, N. Faure, G. Cheng, and R. Stoian, Phys. Rev. B 92, 174109 (2015).

[96] J. Bonse, A. Rosenfeld, and J. Krüger, J. Appl. Phys. 106, 104910 (2009).

[97] F. Garrelie, J.-P. Colombier, F. Pigeon, S. Tonchev, N. Faure, M. Bounhalli, S. Reynaud, and O. Parriaux, Opt. Express 19, 9035 (2011).
[98] G. D. Tsibidis, M. Barberoglou, P. A. Loukakos, E. Stratakis, and C. Fotakis, Phys. Rev. B 86, 115316 (2012).

[99] S. K. Das, H. Messaoudi, A. Debroy, E. McGlynn, and R. Grunwald, Opt. Mater. Express 3, 1705 (2013).

[100] T. J.-Y. Derrien, T. E. Itina, R. Torres, T. Sarnet, and M. Sentis, J. Appl. Phys. 114, 083104 (2013).

[101] M. Rahmani, A. E. Miroshnichenko, D. Y. Lei, B. Luk'yanchuk, M. I. Tribelsky, A. I. Kuznetsov, Y. S. Kivshar, Y. Francescato, V. Giannini, M. Hong, and S. A. Maier, Small 10, 576 (2014).

[102] I. Romero, J. Aizpurua, G. W. Bryant, and F. J. G. D. Abajo, Opt. Express 14, 9988 (2006).

[103] J.-W. Yao, C.-Y. Zhang, H.-Y. Liu, Q.-F. Dai, L.-J. Wu, S. Lan, A. V. Gopal, V. A. Trofimov, and T. M. Lysak, Opt. Express 20, 905 (2012).

[104] C. Hnatovsky, R. S. Taylor, P. P. Rajeev, E. Simova, V. R. Bhardwaj, D. M. Rayner, and P. B. Corkum, Appl. Phys. Lett. 87, 014104 (2005).

[105] E. Bricchi, Ph.D. thesis, University of Southampton, 2005.

[106] K. Mishchik, G. Cheng, G. Huo, I. M. Burakov, C. Mauclair, A. Mermillod-Blondin, A. Rosenfeld, Y. Ouerdane, A. Boukenter, O. Parriaux, and R. Stoian, Opt. Express 18, 24809 (2010).

[107] F. Zimmermann, A. Plech, S. Richter, A. Tünnermann, and S. Nolte, Laser Photon. Rev. 1 (2016). 